\title{
PULSATIONAL INSTABILITY OF ACCRETION DISKS AROUND COMPACT OBJECTS
}

\author{
T. OKUDA \\ Hakodate College, Hokkaido Univ. of Education, Hakodate 040, Japan \\ and \\ S. MINESHIGE \\ Department of Physics, Ioaraki Univ., Mito 310, Japan
}

\begin{abstract}
Linear analysis shows that radial oscillations in accretion disks around compact abject are overstable to axisymmetric perturbation under a variety of conditions. Furthermore, numerical simulations confirm that the radial oscillations induce quasi-periodic modulations of the disk luminosity. The disk oscillation model may be responsible for quasi-periodic oscillations (QPOs) observed in low mass X-ray binaries (LMXBs), cataclysmic variables (CVs), and other compact objects.
\end{abstract}

Pulsational instability of accretion disks around compact objects was first examined by Kato (1978) and later confirmed by Blumenthal, Yang, and Lin (1984) under a variety of conditions. They found that the accretion disks are unstable to axisymmetric radial perturbations and suggested that the radial oscillations with local Keplerian frequencies may explain QPOs in CVs. Papaloizou and Stanley (1986) treated analytically and numerically stability of an axisymmetric accretion disk which includes the disk-boundary layer. Okuda and Mineshige (1991) and Okuda et al. (1992) recently performed numerical simulations of the radial oscillations, with special attention to the QPOs in LMXBs and CVs, respectively.

We summarize these results briefly. Excitation mechanism of the radial oscillations can be understood in analogy with the mechanism in stellar pulsation. In the frame work of $\alpha$-model as to viscous stress, the major thermal energy is supplied by viscous dissipation and the viscous stress $P_{r \phi}$ is assumed to be proportional to local pressure. The viscous energy generation rate increases in the compressed phase during the radial oscillations, leading to amplification of the oscillations.

Our numerical simulations start with initial conditions of the stationary solution by Shakura and Sunyaev (1973) corresponding to an input mass flow rate, $\dot{M}_{0}$, which is kept constant during the calculations. The radial pressure gradient force in the momentum equation, which is neglected in the stationary solution, works as initial small perturbations. The disks are also assumed to be Keplerian both at inner and outer edges.

As far as the input mass flow rate $\dot{M}_{0}$ is low in comparison with the critical accretion rate $\dot{M}_{c}$ corresponding to the Eddington luminosity, the 


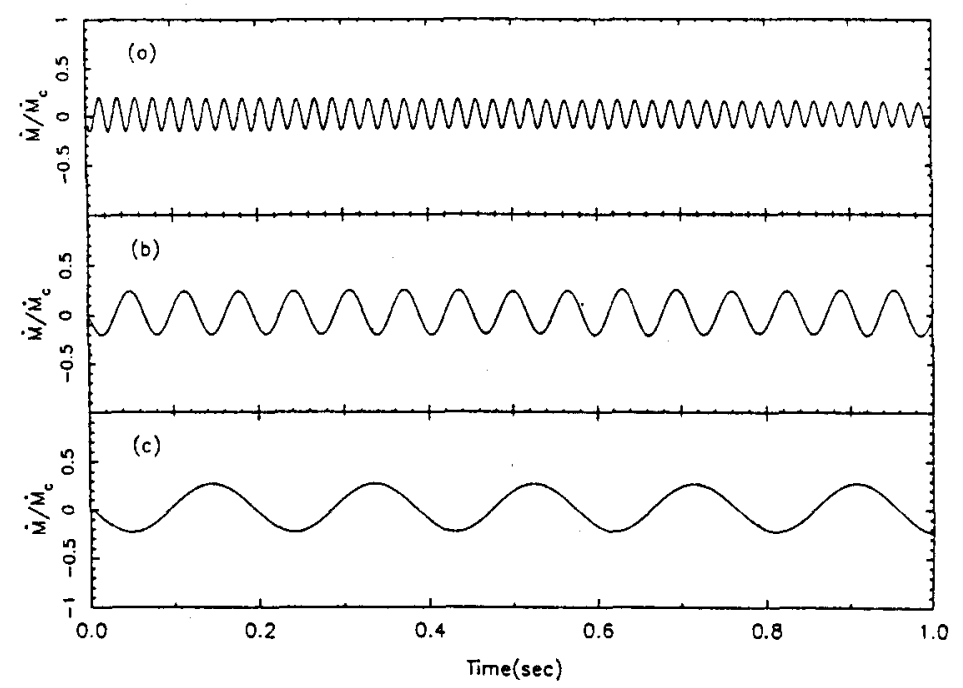

Fig. 1. Modulations of mass flow rate, $\dot{M}$, at $r / r_{\text {in }}=12.9(\mathrm{a}), 27.3(\mathrm{~b}), 56.2$ (c) for a neutron star with $\dot{M}_{0}=0.02 \dot{M}_{c}$ and $\alpha=0.1$.

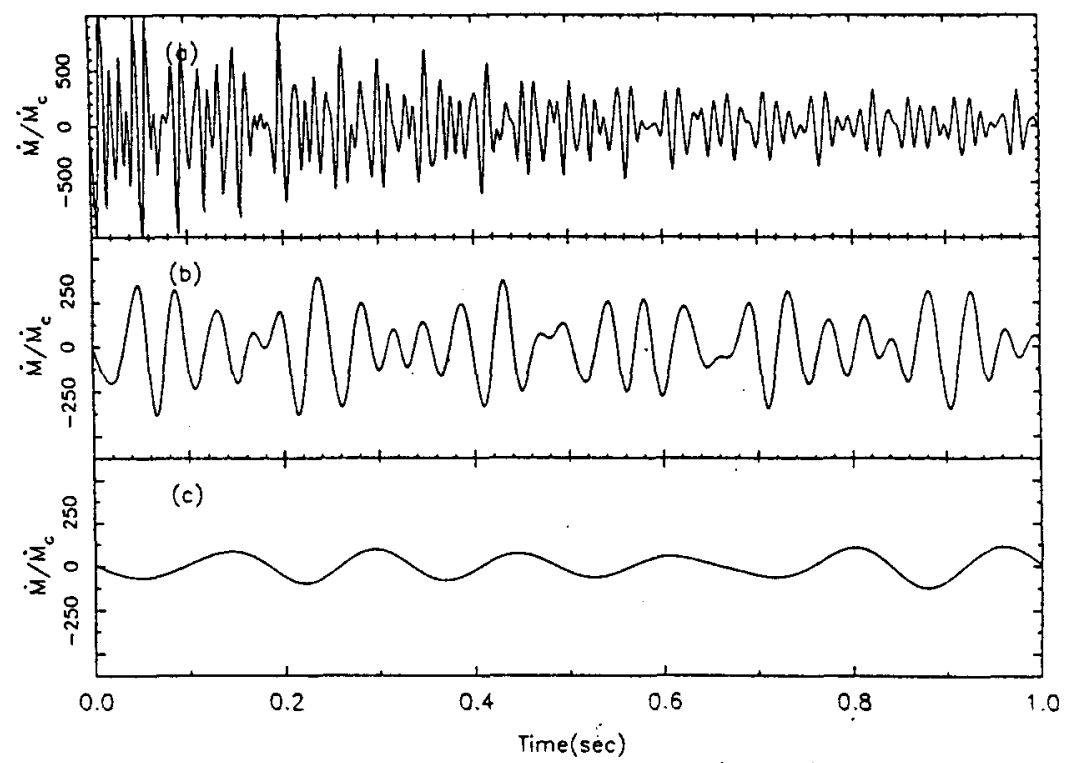

Fig. 2. Same as in Fig. 1 but with $\dot{M}_{0}=\dot{M}_{c}$.

flow variables modulate in good agreement with the result of the linear analysis. Fig. 1 shows modulations of the mass flow rate $\dot{M}$ at $r / r_{\text {in }}=$ 12.9(a), 27.3(b), and 56.2(c) for an accretion disk around a neutron star with $\dot{M}_{0} / \dot{M}_{c}=0.02$ and $\alpha=0.1$, where $r_{\text {in }}$ is the disk inner edge. The mass flow rate shows sinusoidal oscillations with Keplerian frequencies. When $\dot{M}_{0}$ 


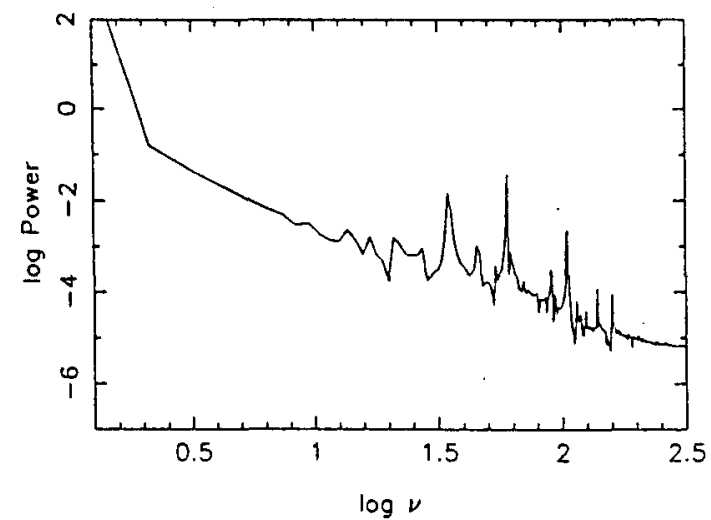

Fig. 3. Power spectra of 1-3 kev disk luminosity oscillation in the same model as Fig. 2.

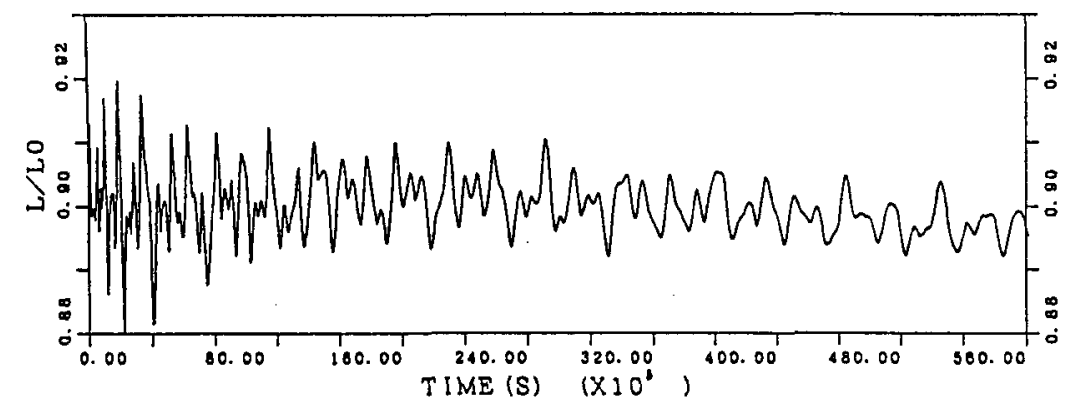

Fig. 4. Disk luminosity modulations for an active galactic nucleus with $M=10^{8} M_{\odot}$, $\dot{M}_{0}=\dot{M}_{c}$, and $\alpha=0.1$.

is so high, overall features of the oscillations deviate from the sinusoidal form and become somewhat chaotic due to nonlinear effect in the disk equations. Fig. 2 depicts the time variation of $\dot{M}$ at three radii for the neutron star with $\dot{M}_{0} / \dot{M}_{c}=1.0$ and $\alpha=0.1$, where the viscous stress $P_{r \phi}=-\alpha P_{g}$ is adopted.

It should be noted that relative oscillation amplitudes of $\dot{M}$ are generally large, whereas oscillation amplitudes of the temperature $T$, the surface density $\Sigma$ and the azimuthal velocity $v_{\phi}$ are as small as $0.1 \sim 10$ percent, depending on the input mass flow rate $\dot{M}_{0}$. Large oscillation amplitudes of the flow variables are numerically found in the region of $r=4 \sim 12 r_{\text {in }}$.

As the results, the total disk luminosity, $L_{d}$, which is an integral of local emergent flux over the whole disk, is modulated quasi-periodically. The modulations are characterized by the power spectra of $L_{d}$, which is shown in Fig. 3. Some discrete peaks are found in the power spectra. This may be a.ttributed to the coarse mesh spacing of the disk model used here. Actual disk 
oscillations are represented as compound of an infinite number of oscillators, each having a different frequency near the Keplerian frequency and a different amplitude. Resultant power spectra should show a more smooth and broader peak (see Okuda et al. 1992). These quasi-periodic light variations are also expected in other class of compact objects such as white dwarfs, black holes, and active galactic nuclei. Fig. 4 shows luminosity modulations for an accretion disk around an active galactic nucleus with $M=10^{8} M_{\odot}$, $\dot{M}_{0}=\dot{M}_{c}$, and $\alpha=0.1$. The disk oscillation model may be promising for the QPOs observed in many species of compact objects.

\section{References}

Blumenthal, G. R., Yang, L. T. and Lin, D. N. C.: 1984, Astrophysical Journal, 287, 774. Kato, S.: 1978, Monthly Notices of the RAS, 185, 629.

Okuda, T. and Mineshige, S.: 1991, Monthly Notices of the RAS, 249, 684.

Okuda, T., Ono, K., Tabata, M. and Mineshige, S.: 1992, Monthly Notices of the RAS, $254,427$.

Papaloizou, J. C. B. and Stanley, G. Q. G.: 1986, Monthly Notices of the RAS, 220, 593.

Shakura, N. I. and Sunyaev, R. A.: 1973, Astronomy and Astrophysics, 24, 337. 